\title{
Genomic survey of SARS-CoV-2 vaccine breakthrough infections in healthcare workers from Kerala, India
}

Beena Philomina J1, ${ }^{1,}$ Bani Jolly2,3, ${ }^{2}$, Neethu John ${ }^{4}$, Rahul C. Bhoyar ${ }^{2}$, Nisha Majeed ${ }^{5}$, Vigneshwar Senthivel2,3, Fairoz C. $P^{1}$, Mercy Rophina ${ }^{2,3}$, Bindhu Vasudevan ${ }^{6}$, Mohamed Imran ${ }^{2,3}$, Prasanth Viswanathan ${ }^{7}$, Arvinden V.R. ${ }^{2,3}$, Anoop Joseph ${ }^{8}$, Mohit Kumar Divakar ${ }^{2,3}$, Priyanka R Nair ${ }^{7}$, Afra Shamnath², P. Jayesh Kumar ${ }^{9}$, Abhinav Jain ${ }^{2,3}, P$. Geetha $^{9}$, Anjali Bajaj2,3, Samatha Mathew ${ }^{2,3}$, Vishu Gupta ${ }^{2,3}$, Srashti Jyoti Agrawal ${ }^{2,3}$, Vinod Scaria, ${ }^{2,3, \#, ~ S r i d h a r ~}$ Sivasubbu ${ }^{2,3, \#, ~ C h a n d n i ~ R a d h a k r i s h n a n ~}{ }^{10 \#}$

\section{Affiliations}

${ }^{1}$ Department of Microbiology, Government Medical College, Kozhikode, Kerala, India

${ }^{2}$ CSIR Institute of Genomics and Integrative Biology (CSIR-IGIB), Mathura Road, New Delhi, India

${ }^{3}$ Academy for Scientific and Innovative Research, Human Resource Development Centre Campus, Ghaziabad,

Uttar Pradesh, India

${ }^{4}$ Department of Microbiology, Government Medical College, Ernakulam, Kerala, India

${ }^{5}$ Department of Microbiology, Government Medical College, Idukki, Kerala, India

${ }^{6}$ Department of Community Medicine, Government Medical College, Ernakulam, Kerala, India

${ }^{7}$ Viral Research and Diagnostic Laboratories, Government Medical College, Kozhikode, Kerala, India

${ }^{8}$ Department of General Medicine, Government Medical College, Ernakulam, Kerala, India

${ }^{9}$ Department of General Medicine, Government Medical College, Kozhikode, Kerala, India

${ }^{10}$ Department of Emergency Medicine, Government Medical College, Kozhikode, Kerala, India

${ }^{*}$ Equal contribution

\#Corresponding author: Chandni R Email: chandnidr@gmail.com Vinod Scaria Email: vinods@igib.in AND Sridhar Sivasubbu, Email: sridhar@igib.in

Keywords: COVID-19, SARS-CoV-2, breakthrough infection, vaccine, whole-genome sequencing. 


\section{ABSTRACT}

We describe genomic analysis of SARS-CoV-2 isolates from breakthrough infections in six healthcare workers following vaccination with AZD1222/Covishield. Four patients were infected by the variant of concern B.1.1.7 while other isolates possessed E484K and S477N mutations in spike protein associated with immune escape.

\section{INTRODUCTION}

The ongoing coronavirus disease 2019 (COVID-19) pandemic has had profound effects across the globe, with multiple waves of infection in many countries caused by waning natural immunity and rise of highly transmissible variants [1]. The tremendous progress in development of vaccines and their deployment is slated to curb recurring waves of COVID-19.

COVID-19 vaccines based on different strategies are widely used across the globe. Of these, inactivated SARS-CoV-2 virus based BBV152/COVAXIN and chimpanzee adenoviral vector based AZD1222/Covishield (ChAdOx1 nCoV-19) are widely used in India[2,3]. Vaccine breakthrough infections in fully vaccinated individuals have been documented in many countries including India [4]. Furthermore, the emergence of SARS-CoV-2 variants with potential immune escape variants threatens the continued efficacy of these vaccines with increasing reports regarding reduced efficacy of vaccines against different SARS-CoV-2 variants of concern (VoC) $[5,6]$.

In this study, we describe the genomic characterization of vaccine breakthrough infections following COVID-19 vaccine in six healthcare workers from South India. The prognosis of the breakthrough infections in all cases show the effective protection of the vaccine in preventing severe COVID-19. We surmise that genomic surveillance is useful to understand and monitor evolving SARS-CoV-2 variants.

\section{METHODS}

\section{Sample Collection}

The RNA extracted from nasopharyngeal swab samples were collected as part of routine COVID-19 testing after informed consent as per the institutional ethical committee guidelines (IHEC-CSIRIGIB/IHEC/2020-21/01) for individuals who had been tested positive following two doses of the AZD1222/Covishield vaccine. Antigen assay (Standard Q Covid-19 Ag Kit, SD Biosensor) was carried out in five out of six patients after they tested RT-PCR positive. Details are in Supplementary Table 1.

\section{Viral Genome Sequencing}

Sequencing ready libraries were prepared from RNA samples using Illumina amplicon based COVIDSeq test [7]. After quality check, all the synthesized libraries were sequenced on NovaSeq 6000 platform with read length of $100 \times 2$ base pairs.

\section{Genome Assembly and Analysis}

The raw FASTQ reads generated by sequencing were trimmed using Trimmomatic (v0.39) and aligned against the Wuhan-Hu-1 reference genome (NC_045512.2) using HISAT2-2.1 to generate a referencebased assembly. Variant calling was done using VarScan (v.2.4). Consensus FASTA sequences for all isolates were generated using samtools (v1.10) and seqtk (v1.3). Genomic variants were identified at a minimum coverage of five reads, and a minimum frequency of $50 \%$.

\section{Phylogenetic Clustering and Visualisation}

Phylogenetic clustering for the genome isolates was done using Nextstrain with additional SARS-CoV-2 genomes isolated from the state of Kerala. Sequences having more than 2000 ambiguous sites in the FASTA file were excluded. Lineages were assigned to the genomes using pangolin (v2.3.9, pangoLEARN version 2021-04-14) [8]. 


\section{RESULTS}

\section{Clinical history and timelines}

A total of six healthcare workers with vaccine breakthrough infections were followed up. All of them had been administered two doses of the AZD1222/Covishield vaccine. The clinical presentations are summarised in Supplementary Table 1.

\section{Patient 1 (P1)}

$\mathrm{P} 1$, a 25-year-old female was administered the first dose of vaccine on January 21, 2021 and the second dose on February 19. P1 was found to be positive for SARS-CoV-2 antigen on March 23, 2021 with influenza-like illness (ILI) symptoms and subsequently tested positive for COVID-19 through RTPCR (Ct cut-off- 37, General Biologicals) on March 25 with Ct values 16.45 and 17.07 for E gene and ORF1ab respectively.

Patient 2 (P2)

P2 was a 50-year-old male who received the first dose of vaccine on January 27, 2021 and the second dose on February 24. He presented with fever, malaise, anosmia and headache on March 31, 2021, following which he tested RT-PCR positive for COVID 19 on April 2 with Ct values 20 for E gene and 19 for ORF1ab. Additionally, P2 was found to be SARS-CoV-2 IgG positive (12.94) and SARS-CoV-2 IgM negative on March 31.

Patient 3 (P3)

P3 was a 53-year-old female who received her first dose of vaccine on January 29, 2021 and the second dose on February 26. She developed rhinitis on March 23, 2021 and tested positive through RT-PCR on March 27 with Ct values 21 for E gene and 19 for ORF1ab.

Patient 4 (P4)

P4 was a 25-year-old female who received the first and second dose of vaccine on February 5 and 10 March, 2021 respectively. She presented with fever, loose stools, abdominal pain, dry cough, severe myalgia, rhinitis and anosmia on March 27, 2021 and tested RT-PCR positive on April 3 with Ct values 24 and 25 for E gene and ORF1ab respectively.

Patient 5 (P5)

P5 was a 32-year-old male who received the first dose of vaccine on January 28,2021 , followed by the second dose on March 12. He tested RT-PCR positive on April 6, 2021 and had mild nasal congestion for a few days before testing, followed by a headache one day after the test. He subsequently tested antigen negative after a period of 10 days.

Patient 6 (P6)

P6, a 33-year-old female, received the first dose of vaccine on January 25, 2021 and second dose on February 22. Initial onset of symptoms including loss of smell, loose stools and rhinitis in P6 was observed on March 9, 2021 and she subsequently tested RT-PCR positive on March 12. P6 tested antigen negative after a period of 5 days. Additionally, neutralizing antibody titres for P6 were found to be above 320 (S/Co value -14.9) as on March 16. Figure 1A summarizes the history and timeline of infection for the 6 patients. All patients were residents of Kerala, a state in southern India.

\section{Genomic variants and lineages}

Genome sequences for the 6 isolates were assembled at mean genome coverage of 7476.27X. Analysis of the genomic variants revealed that 4 samples (P2-P5) had N501Y variant in spike protein, while isolates $\mathrm{P} 1$ and $\mathrm{P} 6$ had $\mathrm{E} 484 \mathrm{~K}$ and $\mathrm{S} 477 \mathrm{~N}$ variants in spike protein respectively. N501Y, E484K 
and $\mathrm{S} 477 \mathrm{~N}$ are key mutations in the receptor binding domain (RBD) of spike protein with substantial evidence reported in the context of immune evasion $[5,9,10]$. The presence of genomic variants in all 6 isolates is summarised in Figure 1B.

Sequencing data indicated that isolate P1 belonged to PANGO lineage B.1.1.306, P6 belonged to lineage B.1.1, while isolates P2, P3, P4, and P5 belonged to the lineage B.1.1.7 (VOC 202012/01). The B.1.1.7 lineage is defined by 6 key genomic variants in the spike protein including N501Y. Phylogenetic context of the 6 viral isolates with 2,630 additional SARS-CoV-2 genome sequences from the state of Kerala in southern India has been summarised in Figure 1C. Isolates P1-P5 clustered closely with other genomes from their respective lineages on the phylogenetic tree and isolate P6 (lineage B.1.1) clustered near genomes belonging to the lineage B.1.560 which was the most prevalent lineage in the 2,630 genomes from Kerala included in the analysis $(\mathrm{N}=1,130)$. 
(A)

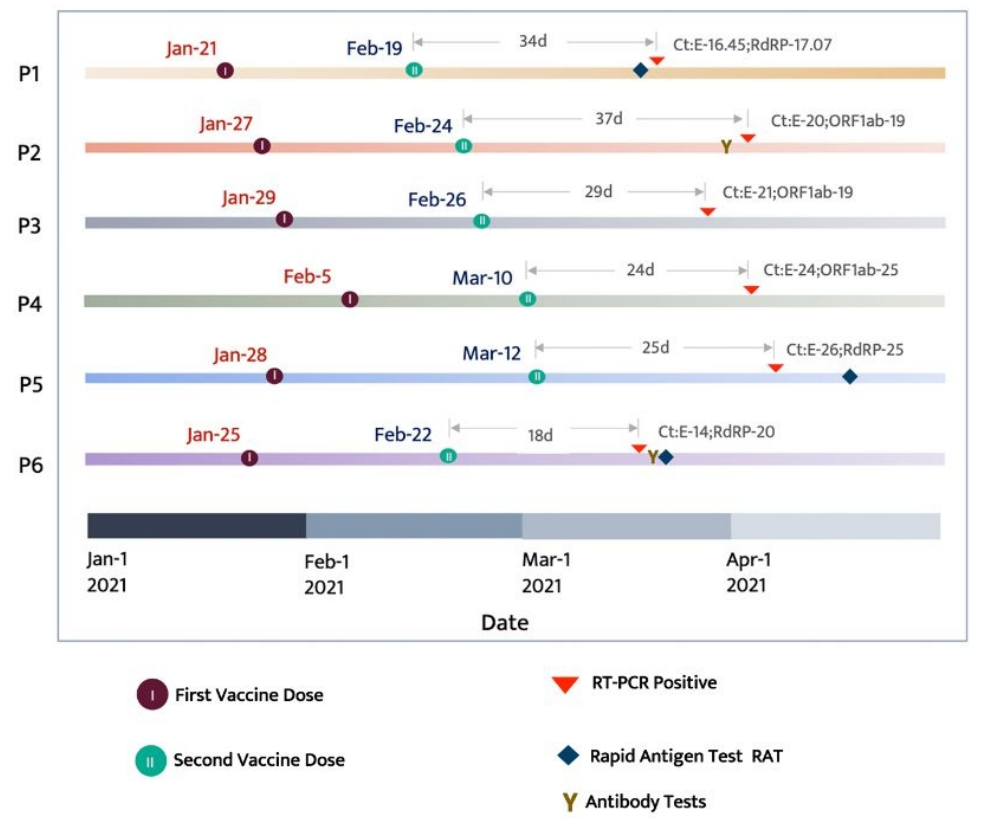

(C)

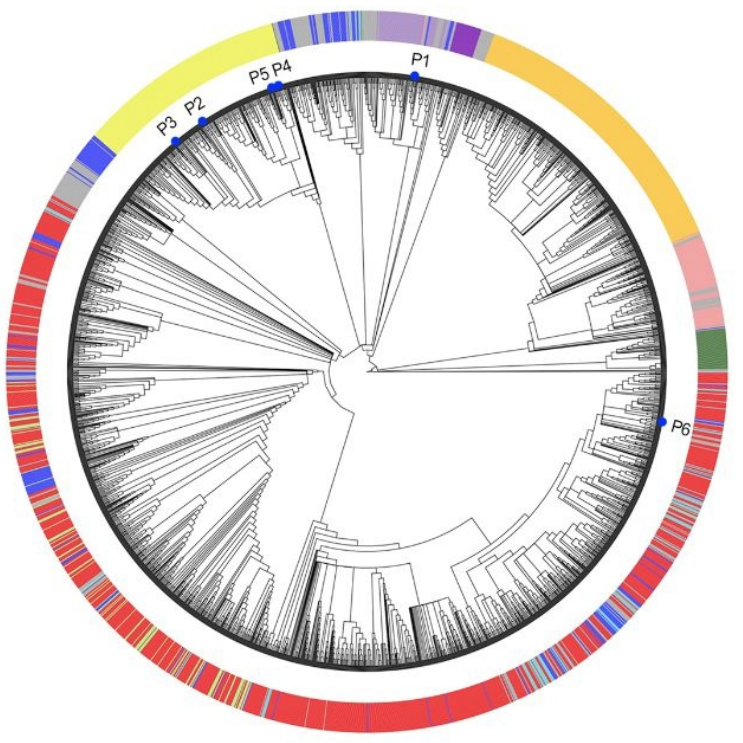

(B)

Patient

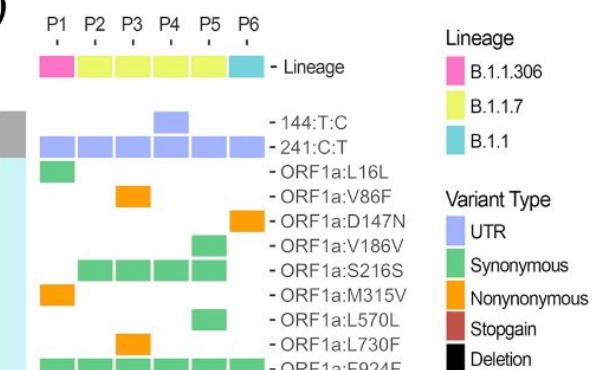

西

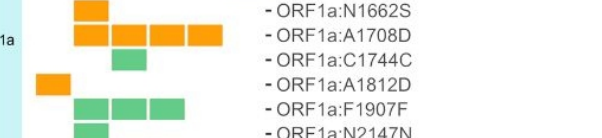




\section{DISCUSSION}

All 6 patients in the study were vaccinated at an interdose interval range of 4-6 weeks and COVID-19 symptoms were observed in all patients at least 15 days post administration of the second dose. Considering that the efficacy against symptomatic infection post 15 days of the second dose of AZD1222 with a 28 day interdose interval is reported as $76 \%$, a small percentage of fully-vaccinated people may still get infected, however it is important to note that none of the 6 patients presented with severe illness [11]. Emerging studies on characterization of clinically important SARS-CoV-2 variants in vaccinated individuals confer possible exploration of the relation between immunization mechanisms and selection of viral escape mutants. In the present study, analysis of the SARS-CoV-2 genome sequences revealed that 4 out of the 6 patients were infected by the B.1.1.7 variant of the virus. N501Y, a key mutation in the B.1.1.7 lineage has been reported to escape neutralization by some monoclonal antibodies (mAbs), while N501Y and E484K, individually and in combination reportedly cause a decrease in neutralization activity in patients vaccinated using Moderna (mRNA-1273) or PfizerBioNTech (BNT162b2) [5,12]. Additionally, the B.1.1.7 variant has been shown to lower neutralising antibody titres against AZD1222 with no significant reduction in clinical efficacy as compared to nonB.1.1.7 variants [6]. Analysis of sequence variants showed the presence of E484K and S477N mutations in spike protein of the viral isolates from P1 and P6 respectively. Both variants are reported to escape neutralization by a range of $m A b s$, while $E 484 \mathrm{~K}$ is also reportedly associated with decrease in the neutralizing activity of convalescent and post-vaccination (BNT162b2) sera $[9,10,12]$. While it remains unclear if these breakthrough infections are related to vaccine efficacy, immune evasion, or other factors, the study highlights the importance of continued genomic surveillance and tracking emergent SARS-CoV-2 variants.

\section{ACKNOWLEDGEMENT}

Authors BJ, MKD, AJ, SM, AB acknowledge research fellowships from Council of Scientific and Industrial Research (CSIR), India. VG and SJA acknowledge research fellowships from the University Grants Commission (UGC), India.

\section{FUNDING}

This work was supported by the National Health Mission Kerala as part of the genomic surveillance and the Council of Scientific and Industrial Research (CSIR), India, through grants CODEST and MLP2005. The funders had no role in the analysis of data, preparation of the manuscript or decision to publish.

\section{POTENTIAL CONFLICT OF INTEREST}

The authors report no potential conflicts of interest.

\section{REFERENCES}

1. Hansen $\mathrm{CH}$, Michlmayr D, Gubbels SM, Mølbak K, Ethelberg S. Assessment of protection against reinfection with SARS-CoV-2 among 4 million PCR-tested individuals in Denmark in 2020: a population-level observational study. Lancet 2021; 397:1204.

2. Ella R, Vadrevu KM, Jogdand $\mathrm{H}$, et al. Safety and immunogenicity of an inactivated SARS-CoV-2 vaccine, BBV152: a double-blind, randomised, phase 1 trial. Lancet Infect Dis 2021; 21:637-646.

3. Voysey M, Clemens SAC, Madhi SA, et al. Safety and efficacy of the ChAdOx1 nCoV-19 vaccine (AZD1222) against SARS-CoV-2: an interim analysis of four randomised controlled trials in Brazil, South Africa, and the UK. Lancet 2021; 397:99-111.

4. Breakthrough COVID19 Infections after Vaccinations in Healthcare and Other Workers in a Chronic Care Medical Facility in New Delhi, India. Diabetes \& Metabolic Syndrome: Clinical Research \& 
Reviews 2021; Available at: http://dx.doi.org/10.1016/j.dsx.2021.05.001. Accessed 9 May 2021.

5. Wang Z, Schmidt F, Weisblum $Y$, et al. mRNA vaccine-elicited antibodies to SARS-CoV-2 and circulating variants. Nature 2021; 592:616-622.

6. Emary KRW, Golubchik T, Aley PK, et al. Efficacy of ChAdOx1 nCoV-19 (AZD1222) vaccine against SARS-CoV-2 variant of concern 202012/01 (B.1.1.7): an exploratory analysis of a randomised controlled trial. Lancet 2021; 397:1351-1362.

7. Bhoyar RC, Jain A, Sehgal P, et al. High throughput detection and genetic epidemiology of SARSCoV-2 using COVIDSeq next-generation sequencing. PLoS One 2021; 16:e0247115.

8. Rambaut A, Holmes EC, O'Toole Á, et al. A dynamic nomenclature proposal for SARS-CoV-2 lineages to assist genomic epidemiology. Nat Microbiol 2020; 5:1403-1407.

9. Liu Z, VanBlargan LA, Bloyet L-M, et al. Identification of SARS-CoV-2 spike mutations that attenuate monoclonal and serum antibody neutralization. Cell Host Microbe 2021; 29:477-488.e4.

10. Jangra S, Ye C, Rathnasinghe R, et al. SARS-CoV-2 spike E484K mutation reduces antibody neutralisation. Lancet Microbe 2021; Available at: http://dx.doi.org/10.1016/S2666-5247(21)000689.

11. Immunization, Vaccines, Biologicals. Interim recommendations for use of the ChAdOx1-S [recombinant] vaccine against COVID-19 (AstraZeneca COVID-19 vaccine AZD1222, SII Covishield, SK Bioscience). World Health Organization, 2021. Available at: https://www.who.int/publications/i/item/WHO-2019-nCoV-vaccines-SAGE_recommendationAZD1222-2021.1. Accessed 9 May 2021.

12. SARS-CoV-2 501Y.V2 variants lack higher infectivity but do have immune escape. Cell 2021; 184:2362-2371.e9. 
Figure 1 (A) History and timelines of infection for the 6 patients and intervals between second dose of vaccine and date of RT-PCR testing (B) Presence of variants in the genome isolates of the 6 cases (P1-P6). (C) Phylogenetic context of the 6 genome isolates with 2,630 additional SARS-CoV-2 genomes from the state of Kerala.

\section{Supplementary Table 1: Detailed clinical history of infection for the 6 patients}

\begin{tabular}{|c|c|c|c|c|}
\hline $\begin{array}{l}\text { Patient } \\
\text { ID }\end{array}$ & $\begin{array}{l}\text { RNA isolation } \\
\text { details }\end{array}$ & $\begin{array}{l}\text { RT-PCR Kit Used (Ct } \\
\text { Values) }\end{array}$ & Antigen Assay & $\begin{array}{l}\text { Neutralising Ab assay } \\
\text { (Value) }\end{array}$ \\
\hline $\mathrm{P} 1$ & $\begin{array}{l}\text { Automated RNA } \\
\text { extraction }\end{array}$ & $\begin{array}{l}\text { General Biologicals } \\
\text { (E:16.45, } \\
\text { ORF1ab:17.07) }\end{array}$ & POSITIVE & NOT DONE \\
\hline P2 & $\begin{array}{l}\text { Automated RNA } \\
\text { extraction }\end{array}$ & $\begin{array}{l}\text { General Biologicals } \\
(\mathrm{E}: 20, \text { ORF1ab:19) }\end{array}$ & NOT DONE & $\begin{array}{l}\text { SARS-CoV-2 IgG - Positive } \\
(12.94 / 1) \\
\text { SARS-CoV-2 IgM - Negative } \\
(0.48 / 1)\end{array}$ \\
\hline P3 & $\begin{array}{l}\text { Automated RNA } \\
\text { extraction }\end{array}$ & $\begin{array}{l}\text { General Biologicals } \\
(\mathrm{E}: 21, \text { ORF1ab:19) }\end{array}$ & POSITIVE & NOT DONE \\
\hline P4 & $\begin{array}{l}\text { Automated RNA } \\
\text { extraction }\end{array}$ & $\begin{array}{l}\text { General Biologicals } \\
(E: 24, \text { ORF1ab:25) }\end{array}$ & POSITIVE & NOT DONE \\
\hline P5 & $\begin{array}{l}\text { Automated RNA } \\
\text { extraction }\end{array}$ & $\begin{array}{l}\text { General Biologicals } \\
(E: 26, \text { ORF1ab:25) }\end{array}$ & $\begin{array}{l}\text { Negative (After } \\
\text { RT-PCR } \\
\text { positive) }\end{array}$ & NOT DONE \\
\hline P6 & $\begin{array}{l}\text { Automated RNA } \\
\text { extraction }\end{array}$ & $\begin{array}{l}\text { General Biologicals } \\
(\mathrm{E}: 14, \text { ORF1ab:20) }\end{array}$ & $\begin{array}{l}\text { Negative (After } \\
\text { RT-PCR } \\
\text { positive) }\end{array}$ & $\begin{array}{l}\text { CLIA vitros Antibody Test } \\
\text { (Neutralising Ab titer 14.9) }\end{array}$ \\
\hline
\end{tabular}

